\title{
Évaluation de la rentabilité et de la productivité totale des facteurs de production des exploitations céréalières : cas de la culture du blé dur au Nord de la Tunisie
}

\author{
Zouhair Rached *, Ali Chebil**, Sonia Boudiche***, Raoudha Khaldi*
}

DOI: $10.30682 / \mathrm{nm} 2102 \mathrm{~h}$

JEL codes: Q10, Q54

\begin{abstract}
The main objectives of this paper are to assess the production costs and to measure the total factor productivity (TFP) of durum wheat production for a sample of cereal farmers in the North of Tunisia. Our hypothesis is that improvement of these indicators can reduce the production costs and consequently the improvement of producers' incomes. To test this hypothesis, a survey has been carried out over a sample of 232 cereal farms in the subhumid region in the North of Tunisia cultivating durum wheat. The reconstituted costs approach was used to estimate the production costs. The obtained results show that production costs and net margins of durum wheat vary according to the farm size. About $67 \%$ of surveyed farms make a positive gross margin. The empirical results of the regression reveal that TFP is positively affected by the farm size and the crop rotations, it is negatively affected by the new varieties and the investments made by the farm while.
\end{abstract}

Keywords: Production costs, Gross margin, Total Factor Productivity, Durum wheat, Tunisia.

\section{Introduction générale}

En Tunisie, la céréaliculture constitue la base de la sécurité alimentaire de la population. Toutefois, ce secteur est soumis à plusieurs contraintes économiques, financières et sociales auxquelles s'ajoutent les aléas climatiques et la limitation et la dégradation des ressources naturelles. Il reste le plus concerné par ces défis en raison de son importance économique et sociale et sa contribution à la sécurité alimentaire.
Sur le plan économique, la céréaliculture contribue à raison de $10 \%$ en moyenne à la valeur de la production agricole sur la période 2010-2015 (MARHP, 2015). Ce niveau représente presque le double de celui de la pêche. Il est proche de celui du maraichage et représente le tiers de l'arboriculture et de l'élevage. La céréaliculture occupe une part importante, de l'ordre de 33\% de la Superficie Agricole Utile (SAU). Elle alimente la chaîne de transformation de l'industrie agroalimentaire à travers les

\footnotetext{
* Institut National de la Recherche Agronomique de Tunisie (INRAT), Université de Carthage, Ariana, Tunisie.

** Institut National de la Recherche en Génie Rural, Eaux et Forêts Tunisie (INRGREF), Ariana, Tunisie.

*** École Supérieure des Industries Alimentaires de Tunis (ESIAT), Tunis, Tunisie.

Corresponding author: rachedzouhair1@gmail.com
} 
minoteries et les semouleries et assure une part importante de l'alimentation du bétail.

Sur le plan social, ce secteur joue un rôle important dans la fixation de la population rurale et la préservation de l'emploi agricole. La céréaliculture offre annuellement 2,5 millions de journées de travail, soit 9\% de l'emploi agricole total (INS, 2015). Le nombre des exploitants céréaliers est estimé à $240000(50 \%$ du nombre total des exploitants) dont 104000 se situent dans le nord de la Tunisie.

Les céréales ont toujours été considérées comme des produits stratégiques puisqu'elles constituent la base du régime alimentaire du consommateur tunisien en lui procurant $50,6 \%$ des calories totales et $52 \%$ des protéines totales (INS, 2015). Selon l'enquête de 2015 de l'Institut National de la Statistique (INS), le tunisien reste un grand consommateur de céréales avec une moyenne de $407 \mathrm{~g} /$ personne/jour de blé contre une consommation mondiale moyenne de $178 \mathrm{~g} /$ personne/jour (FAOSTAT, 2013). Le blé dur reste le plus consommé par rapport au blé tendre sous formes de pâtes alimentaires et semoules. Les dépenses en ces produits représentent $16 \%$ des dépenses alimentaires des ménages. (INS, 2015).

Face à son importance stratégique pour la sécurité alimentaire et à son déficit chronique, dans un contexte économique marqué par l'augmentation des prix internationaux, la stratégie céréalière mise en place par les pouvoirs publics est orientée vers l'encouragement et le soutien au développement de la production locale. Ainsi, une série de mesures a été prise en 2008, concernant la production et portant sur la révision des prix à la production de tous les produits céréaliers, le maintien des prix des semences sélectionnées aux mêmes niveaux que ceux de 2007, l'accès à une première irrigation gratuite, l'effacement et le rééchelonnement des dettes des céréaliculteurs (l'effacement des dettes issues des crédits dont le montant ne dépasse pas $2000 \mathrm{DT}^{1}$ en 2002). Ce même programme est encore maintenu au niveau du Ministère de l'Agriculture pour effacer et rééchelonner d'autres dettes qui persistent jusqu'à 2013 (arrêté combiné entre le Ministre de l'Agriculture et le Ministre des Finances concernant la clarification de la loi des finances complémentaires de 2016) avec l'augmentation des superficies irriguées des céréales, qui ont atteint 120000 hectares en 2014, et l'octroi des subventions pour les intrants nécessaires à l'entretien des cultures céréalières irriguées (eau d'irrigation, engrais, pesticides, etc.).Toutes ces mesures ont pour objectif d'atteindre une production permettant de couvrir $60 \%$ des besoins en céréales. Le présent travail contribue à l'analyse de la performance économique des exploitations céréalières en vue d'une durabilité économique des exploitations.

En effet, le coût de production constitue un élément pertinent pour l'analyse de la rentabilité d'une culture. Il a beaucoup intéressé les économistes (Desebois, 2005 ; Boughanmi, 1995). Il nous donne une idée sur les charges, les coûts des facteurs de production et conçoit un élément comparatif entre les spéculations. En outre cet élément est consolidé par le résultat provenant de la synergie des facteurs de production.

Ainsi, la productivité totale des facteurs de production ou la productivité partielle des facteurs a toujours intéressé les politiques agricoles en Tunisie. En effet, l'objectif général des plans de développement agricoles ont toujours été d'améliorer la productivité du secteur agricole et notamment du secteur céréalier.

L'objectif de notre recherche s'intéresse à l'analyse des coûts et leur structure par type d'exploitation en vue de dégager les exploitations au-dessous du seuil de rentabilité et pour lesquelles seront identifiés les déterminants de la productivité globale des facteurs de production.

Ce travail est structuré en trois parties la première porte sur les données économiques relatives au secteur céréalier. La seconde présente le matériel et les méthodes. La troisième est consacrée à la présentation des résultats et des discussions. La conclusion générale fera l'objet de la dernière partie.

$1 \quad 1$ Dinar Tunisien $(\mathrm{DT})=3,3$ Euro $=2,8$ Dollars. 


\section{Situation économique du secteur céréalier}

Les cours mondiaux des céréales, durant les dernières années, ont connu une hausse considérable ayant atteint son maximum en 2007, soit $30 \%$ par rapport à 2006 . Ce phénomène est surtout lié à : (i) la réduction de l'offre mondiale en raison des mauvaises conditions climatiques dans les grands pays exportateurs tels que l'Australie ; (ii) la diminution des stocks de sécurité des pays développés, où les stocks céréaliers sont tombés à leurs niveaux les plus bas depuis les années 80 ; (iii) la flambée des cours du pétrole qui a causé une augmentation des prix des intrants agricoles et des services, à savoir les frais de transport, de transformation et d'entreposage ; (iv) l'augmentation continue de la demande expliquée par les besoins accrus de la fabrication de biocarburants, surtout par les pays exportateurs (USA, Argentine, Ukraine) ; (v) la croissance démographique et l'augmentation durable des revenus des pays émergents tel que la Chine, l'Inde et d'autres économies en développement.
Cette conjoncture internationale a eu des impacts sur la facture des importations des céréales pour la Tunisie. Les prix d'importation des céréales sont passés pour le blé dur, le blé tendre et l'orge respectivement de $160 \mathrm{DT} / \mathrm{t}, 120 \mathrm{DT} / \mathrm{t}$ et $106 \mathrm{DT} / \mathrm{t}$ en 1984 à $554 \mathrm{DT} / \mathrm{t}, 368 \mathrm{DT} / \mathrm{t}$ et 337DT/t en 2007 pour atteindre durant le dernier quinquennat (2012-2016) 708DT/t en moyenne pour le blé dur $460 \mathrm{DT} / \mathrm{t}$ pour le blé tendre et 436 $\mathrm{DT} / \mathrm{t}$ pour l'orge. Ces prix ont enregistré, depuis 2006 un accroissement moyen de $200 \%$ pour le blé dur, $78 \%$ pour le blé tendre et de $83 \%$ pour l'orge dont l'écart le plus important est observé pour le blé dur (Figure 1).

En comparant les prix à la production à celui de l'importation, on remarque qu'à partir de l'année 2007 le prix à la production est nettement inférieur au prix à l'importation, malgré la prime exceptionnelle de livraison avant la fin de la campagne agricole et qui a fait augmenter le prix à la production depuis 2009. De ceci, il découle que produire plus devient une priorité non seulement pour la réalisation de la sécurité alimentaire, mais aussi pour un intérêt économique.

Figure 1 - Évolution des indices de prix à l'importation des céréales les plus importantes (1984-2016) en DT/t (base 100 en 1984).

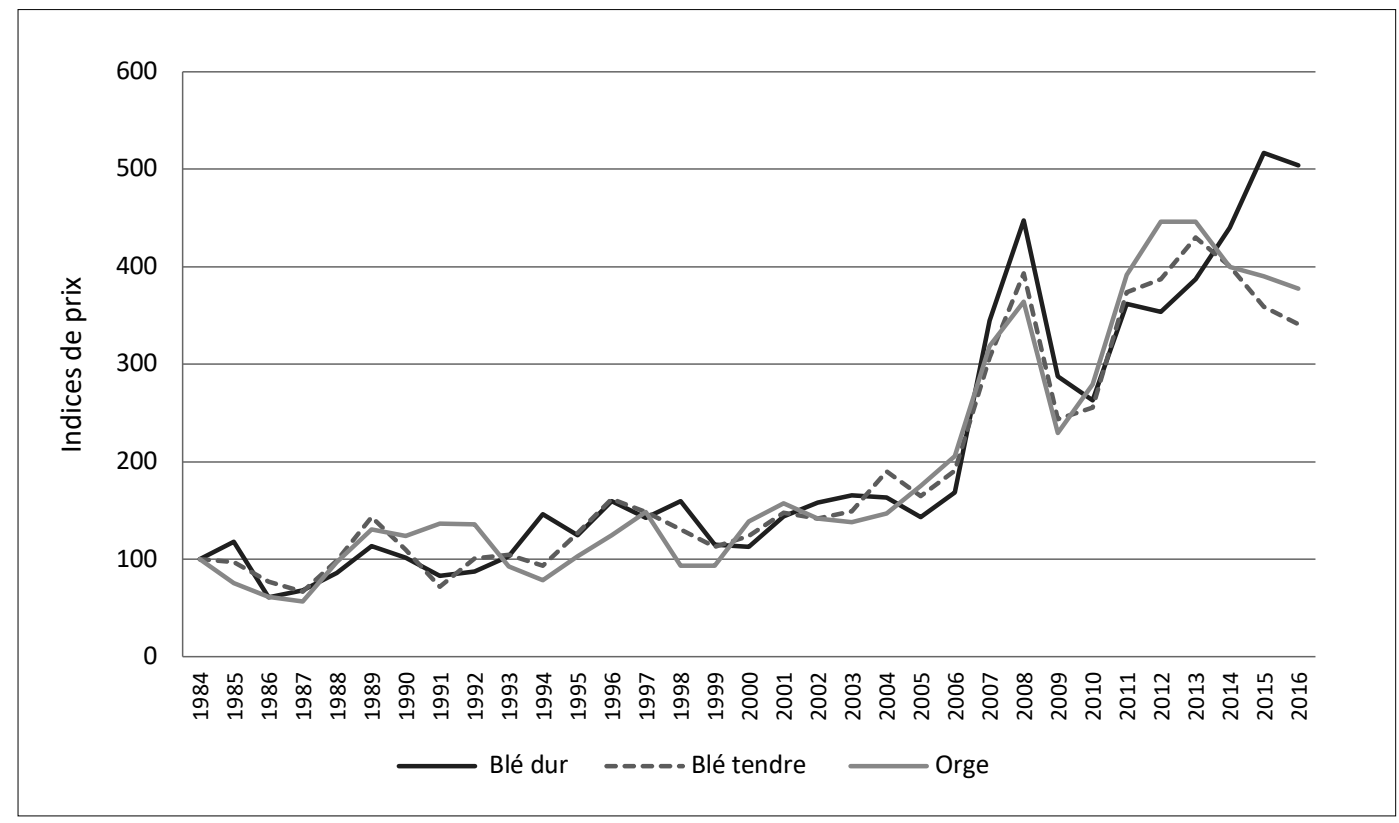

Source : MARHP, 2016. 
Figure 2 - Différence du prix à l'importation et du prix à la production des principales céréales en DT/t (base 100 en 1984).

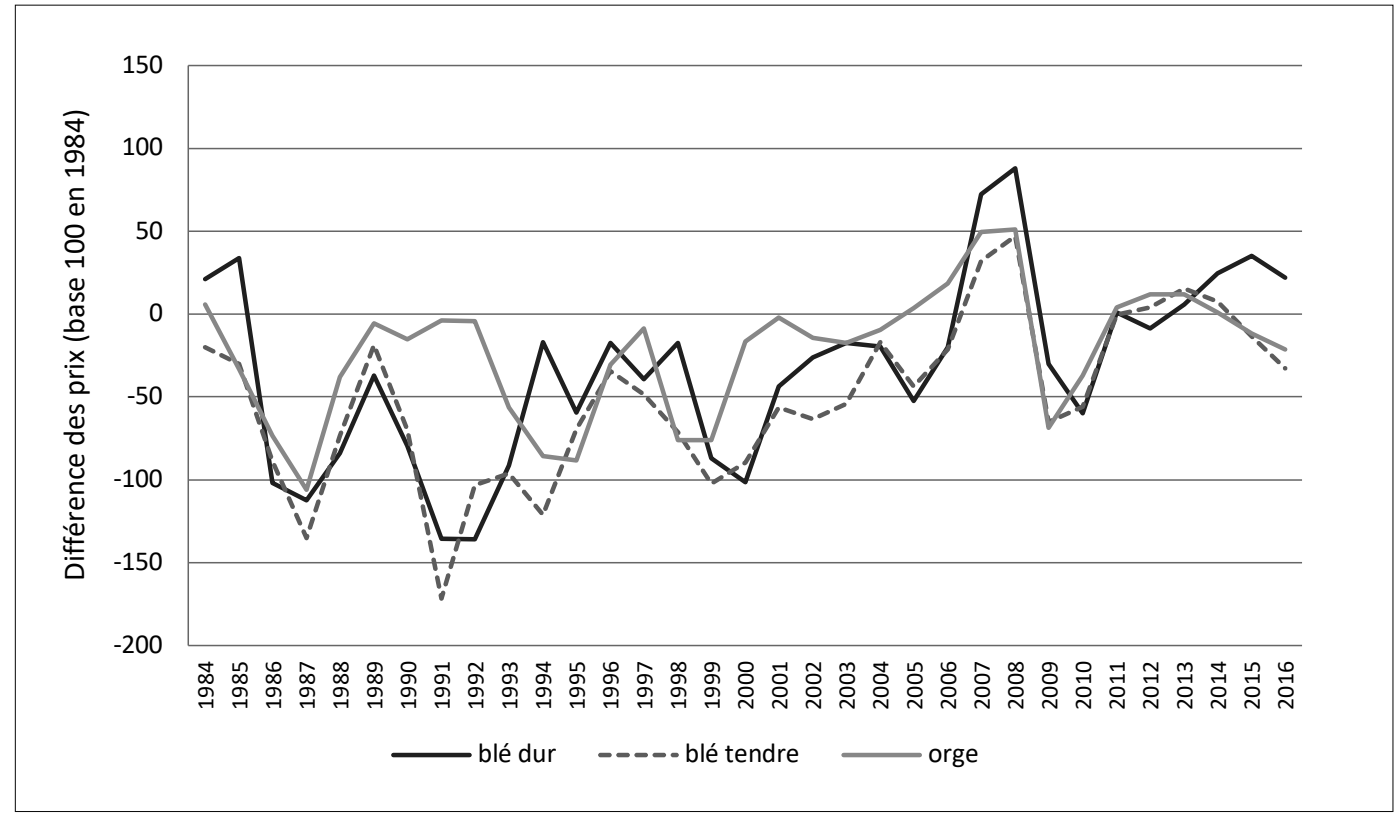

Source : Office des céréales, MARHP, 2016.

Figure 3 - Évolution des importations des principales céréales en Tunisie (1984-2016) (base100 en 1984).

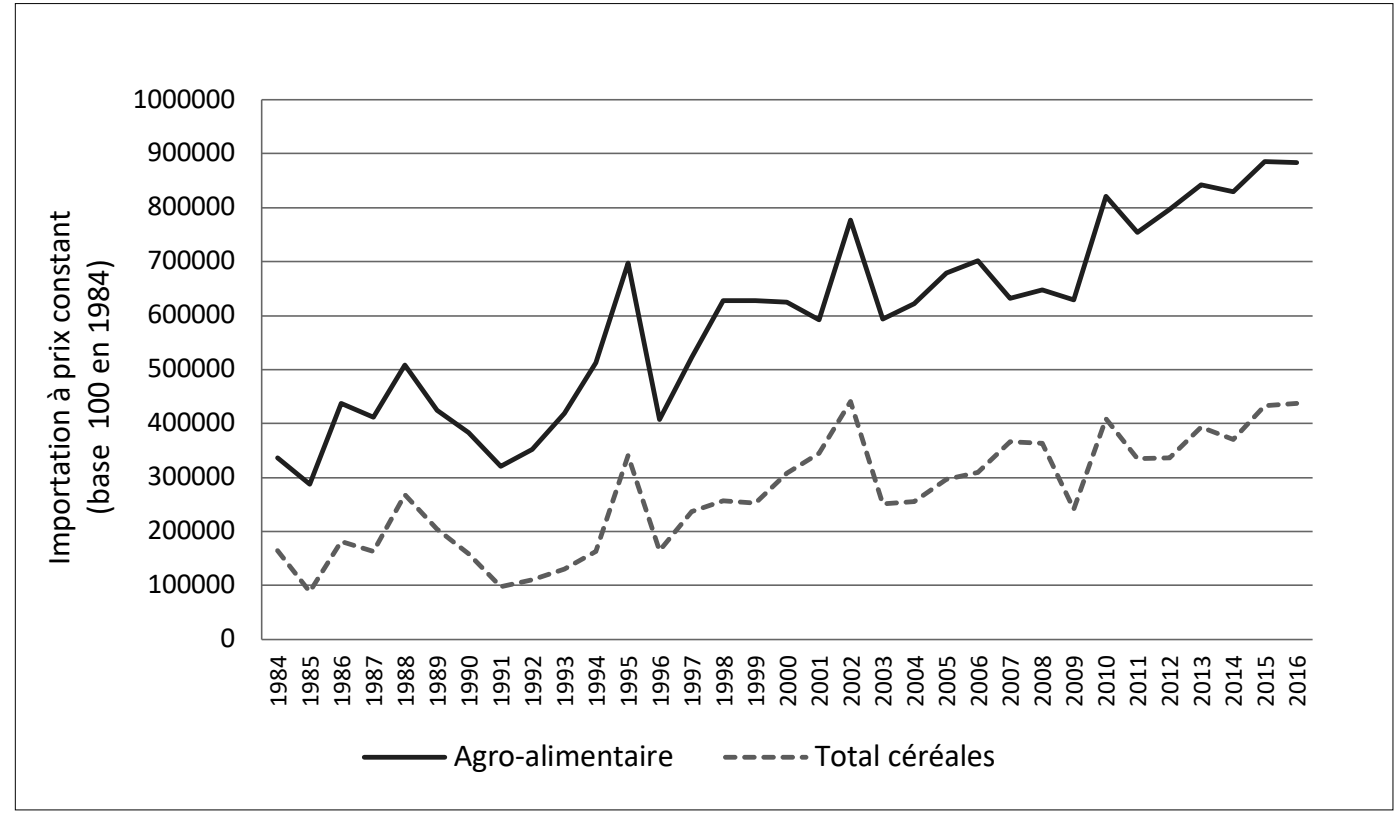

Source : MARHP, 2016. 
Sous l'effet de cette flambée des prix des céréales sur le marché mondial en 2007, la facture des importations des céréales est devenue de plus en plus lourde dans la mesure où sa valeur est passée de 433MDT en 2006 à 960MDT en 2007 pour atteindre 1416MDT en 2015 (INS, 2015). Parallèlement, les charges de la Caisse Générale de Compensation sont passées de 250MDT en 2006 à 520MDT en 2007 pour atteindre 800MDT en 2008. Face à cette conjoncture, il est impératif de réduire l'effet de la flambée des prix mondiaux sur l'équilibre de la balance commerciale en réduisant le déficit céréalier par l'amélioration de la production nationale des céréales (Figure 2). Ce déficit, induit par les facteurs internes et externes, est de plus en plus difficile à supporter. En effet, les difficultés internes se trouvent majorées par une volatilité des cours mondiaux des céréales due notamment aux changements climatiques mais aussi à des comportements spéculatifs des céréaliculteurs.

En plus de la fluctuation des prix des céréales sur le marché mondial, l'offre internationale n'arrive plus à répondre à la demande. Le risque de pénurie surtout pour le blé existe du fait que l'excédent de la production mondiale en ce produit n'a pas dépassé $7 \%$ de sa demande globale durant les cinq dernières années (FAO, 2020).

Compte tenu de ce constat complexe du déficit de la balance commerciale lié aux importations des céréales à des facteurs internes et externes, il est impératif non seulement d'accroitre la production pour assurer la sécurité alimentaire de la population mais aussi de produire avec des prix compétitifs pour des intérêts micro et macro-économiques permettant aux agriculteurs de surmonter la fluctuation des prix des intrants afin de réduire les coûts de production et d'améliorer leurs revenus.

\section{Matériels et méthodes}

\subsection{Le calcul des coûts de production}

Le calcul des coûts de production peut être principalement abordée de deux manières, le coût de production constaté (ou coût réel de production) utilisé par Karray et al. (2000), Khaldi et Zekri (2002) et Rached (2008) et le coût re- constitué (ou coût au prix de marché) Desbois (2006). Dans notre cas, vu la complexité du système des prix d'une part, et la diversité des systèmes de production d'autre part, nous avons choisi l'approche du coût reconstitué pour calculer le coût de production du blé dur pour les différents systèmes de production.

Cette approche normative du coût fait, en grande partie, abstraction des autres productions de l'exploitation et de la réalité des moyens de production présents (parc de matériel et main-d'œuvre familiale). Pour le coût reconstitué, on part des besoins de la culture (en produits, en matériel, en main-d'œuvre, ...). Tout est calculé comme si les travaux étaient « conduits par une autre entreprise privée ou service extérieur ». Le matériel et la main-d'œuvre ne sont pris en compte que pour leur durée d'utilisation. Nous supposons donc que tous les facteurs sont variables et ont des prix sur le marché. Les prix des consommations intermédiaires et celui du prix du marché dans la région, le prix de la mécanisation est celui du prix de location par heure de travail. Pour les prix des trois autres facteurs (capital, travail familial, terre) ils correspondent à des coûts d'opportunité (Bachta et al., 2001). La main-d'œuvre familiale présente sur l'exploitation est assimilée au prix de la main-d'œuvre occasionnelle. Ainsi le prix de la terre correspond au loyer pratiqué dans chaque région en 2019 (Albouchi, 2006). Il est évident que ce loyer dépend des potentialités des régions en question et du type de sol. En ce qui concerne le prix du capital, il correspond au coût de son usage. Théoriquement, le coût d'usage du capital est égal au taux d'amortissement plus le taux d'intérêt. Cependant, pour simplifier le calcul et vue la complexité d'affectation des charges de l'amortissement, le taux d'intérêt est considéré comme prix du capital. Cette approche fournit ainsi un coût de production relativement optimisé et surtout représentatif du système de production correspondant à des grandes étendues homogènes.

\subsection{Calcul de la marge brute}

Le calcul du coût de production reconstitué est la somme des charges totales (CHT) correspondantes. C'est aussi les charges fixes (CHF) plus les charges variables (CHV). Les charges variables sont les charges qui varient en fonc- 
tion de la quantité produite. Elles représentent la valeur des quantités des intrants, de la mécanisation, de la main d'œuvre et du capital investi. Les charges fixes dans notre cas, sont les charges de l'assurance, la valeur locative de la terre et les charges financières. Les charges totales de production sont calculées à partir de la formule suivante :

$$
C H T=\sum_{i=1}^{n} w v_{i} * q v_{i}+\sum_{i=1}^{n} w f_{i} * q f_{i}
$$

Le coût unitaire (CU) de production est calculé par la soustraction de la valeur des sous produits divisé par la quantité produite. Les sous produits pour la culture céréalière sont la paille. Selon Gharbi et al. (2002), le calcul du coût unitaire est estimé par la formule suivante :

$$
\begin{gathered}
C U=\left[\left(\sum_{i=1}^{n} w v_{i} * q v_{i}+\right.\right. \\
\left.\left.\sum_{i=1}^{n} w f_{i} * q f_{i}\right)-\mathrm{Qs} * \mathrm{ps}\right] / \mathrm{Q}
\end{gathered}
$$

Avec :

- $\mathrm{qf}_{\mathrm{i}}$ et $\mathrm{wf}_{\mathrm{i}}$ sont respectivement les quantités et les prix des facteurs (charges) fixes pour la production du blé dur ;

- $\mathrm{wv}_{\mathrm{i}}$ et $\mathrm{qv}_{\mathrm{i}}$ représentent respectivement les prix et les quantités des facteurs spécifiques pour la production de blé dur;

- ps et Qs sont le prix et la quantité de sous produit respectivement.

La marge brute $(\mathrm{MB})$ est la différence entre le produit brut et les charges spécifiques pour la production du blé dur celle-ci est calculée par la formule suivante :

$$
\mathrm{MB}=\mathrm{P} * \mathrm{Q}-\sum_{\mathrm{i}=1}^{\mathrm{n}} \mathrm{wvi} * \mathrm{qv}_{\mathrm{i}}
$$

dont : P et $\mathrm{Q}$ sont respectivement le prix et la quantité produite de blé dur, produit par l'exploitation.

\subsection{Productivité totale des facteurs de production}

La productivité totale des facteurs de production (PTF) a été estimée par le rapport entre la production totale et la somme des charges variables estimé par l'approche des coûts reconstitués. Selon Bamidele et al. (2008), Mbam et Edeh (2011), Dhehibi et al. (2012), la productivité est estimée par la formule suivante :

$$
\mathrm{PTF}_{j}=\frac{\mathrm{R}_{\mathrm{j}}}{\sum_{\mathrm{i}=1}^{\mathrm{n}}\left(\mathrm{CVT}_{\mathrm{i}}\right)}
$$

dont :

- $\mathrm{PTF}_{\mathrm{j}}$ est la productivité totale des facteurs pour l'exploitation $\mathrm{j}$;

- $\mathrm{R}_{\mathrm{j}}(\mathrm{DT} / \mathrm{ha})$ représente la valeur de la production du blé dur de l'exploitation $\mathrm{j}$;

- CVT (DT/ha) indique le totale des charges variables.

Les déterminants techniques et socio-économiques $\left(\operatorname{det}_{\mathrm{i}}\right)$ de la PTF ont été identifiés par un modèle Adhoc exprimant la PTF en fonction des variables socioéconomiques et techniques. Les variables utilisées (det $t_{\mathrm{i}}$ ) sont la taille de l'exploitation, la rotation des cultures, les variétés de blé dur utilisées pour la production (Slama et al., 2005), la qualité des semences employées, la réalisation des investissements et le morcellement (Dhehibi et al., 2012 ; Chebil et al., 2015 ; Grami et Ben Rejeb, 2015). La relation est la suivante :

$$
\mathrm{PTF}_{\mathrm{i}}=\text { cte }+\sum \theta_{\mathrm{i}} \text { det }_{\mathrm{i}}
$$

Avec det $_{\mathrm{i}}$ : sont les déterminants de la productivité totale des facteurs qui expliquent la variation de la PTF de l'exploitation i.

\subsection{Source des données}

Les données primaires utilisées sont collectées en 2010-2011 à partir des enquêtes par questionnaire pluridisciplinaire auprès de 232 céréaliculteurs produisant du blé dur au cours de la campagne 2009-2010. Ces producteurs enquêtés sont repartis sur trois gouvernorats appartenant à l'étage bioclimatique subhumide à savoir Beja, Jendouba et Bizerte. Le choix de ces gouvernorats a été réalisé en fonction de leurs contributions, en termes de production et de superficie, dans l'offre nationale de blé dur et du nombre de céréaliculteurs actifs. La méthode d'échantillonnage en grappes stratifiées à trois niveaux a été retenue $\left(1^{\text {er }}\right.$ niveau : gouvernorat, $2^{\text {ème }}$ niveau : délégation, $3^{\text {ème }}$ niveau : taille de l'exploitation). Le nombre des céréaliers par région a été identifié en fonction de la population mère de chaque gouvernorat, soit $0,3 \%$ des producteurs du blé dur de chaque gouvernorat et ce compte tenu des moyens disponibles. Ensuite, on a sélectionné les délégations appartenant à l'étage bioclimatique subhumide au sein de chaque gou- 
vernorat. Puis dans ces délégations, la taille de l'exploitation (strate de superficie) a été retenue comme facteur déterminant de l'exploitant à enquêter, soit $32 \%$ des petites exploitations, $30 \%$ des moyennes exploitations $(17 \%$ de 5 à 10 ha et $11 \%$ de 10 à 50 ha) et $36,6 \%$ des grandes exploitations (plus de 50 ha). Enfin, le choix des exploitants de chaque strate a été réalisé d'une façon aléatoire simple à probabilité égale soit de $0,3 \%$ de la population mère de producteurs du blé dur, en assurant une répartition équitable entre les grandes, les moyennes et les petites exploitations céréalières soit près de $33 \%$ des agriculteurs de chaque catégorie. Notre recherche ne vise pas la représentativité des exploitations plus que la diversité de leurs systèmes de production.

\subsection{Présentation et choix de la zone d'étude}

Les gouvernorats de Béja, Jendouba et Bizerte se situent au Nord de la Tunisie. Ils sont limités au Nord par la Méditerranée, à l'Ouest par l'Algérie, au Sud par les gouvernorats du Kef, Siliana, Zaghouan et à l'Est par la Mer Méditerranée et le gouvernorat de l'Ariana (Figure 4). Cette zone dispose d'une superficie totale de l'ordre de $10527 \mathrm{~km}^{2}$.

En plus la zone subhumide est la plus favorable pour la culture céréalière, notamment le blé dur. Elle est caractérisée par une pluviométrie variante entre 500 et $700 \mathrm{~mm} / \mathrm{an}$ (voir 1200 $\mathrm{mm}$ à Fernana). Il est à noter aussi qu'elle est la plus ancienne zone productrice de blé. En termes de superficie, ces gouvernorats représentent $13 \%$ de la superficie agricole utile. La céréaliculture y occupe près de $50 \%$ de la superficie labourable représentant $20 \%$ de la superficie céréalière totale du pays. La culture du blé dur accapare à
Figure 4 - Plan de situation de la zone d'étude.

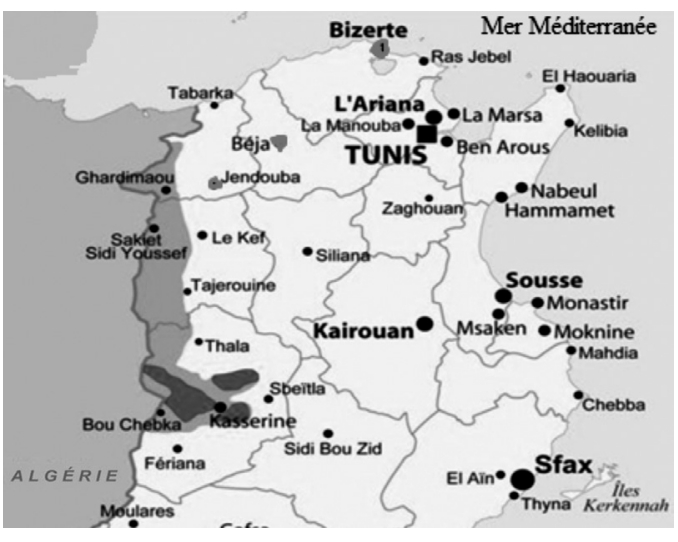

elle seule $70 \%$ de la superficie céréalière de la région qui contribue à raison de $46 \%$ à la production totale de blé dur (MARHP, 2015).

Selon l'enquête réalisée sur les céréales en 2012 par le Ministère de l'Agriculture, des Ressources Hydrauliques et de la Pêche, le Nord de la Tunisie est le principal fournisseur de céréales. Il produit $78 \%$ des céréales. Les régions de Bizerte, Beja et Jendouba contribuent dans la production nationale céréalière avec une part de $10 \%, 16 \%$ et $9 \%$ respectivement. En total, ces régions fournissent $46 \%$ de la production nationale du blé dur (Tableau 1).

\section{Résultats et discussions}

\subsection{Charges de production du blé dur}

Le Tableau 2 montre les valeurs moyennes, minimales et maximales ainsi que les écarts types des charges variables pour l'ensemble des exploitations et par taille d'exploitation. Les charges variables par hectare varient pour le total de l'échan-

Tableau 1 - Superficie des céréales par région de production.

\begin{tabular}{|lcccc|}
\hline & Béja & Bizerte & Jendouba & Total Tunisie \\
\hline Superficie céréales (1000ha) & 144 & 92 & 82 & 1610 \\
\hline Total céréales (\%) & 16 & 10 & 9 & 100 \\
\hline Superficie céréales/ Superficie labourable (\%) & 56 & 43 & 49 & 33 \\
\hline Superficie blé dur (1000ha) & 93,1 & 64,6 & 60,6 & 858 \\
\hline Superficie blé dur/Superficie céréales (\%) & 65 & 70 & 74 & 53 \\
\hline
\end{tabular}

Source : Enquête sur les structures MARHP, 2012. 
Tableau 2 - Charges variables pour la production du blé dur.

\begin{tabular}{|c|c|c|c|c|c|c|c|c|}
\hline & & $\begin{array}{c}\text { Mécani- } \\
\text { sation } \\
(D T / h a)\end{array}$ & $\begin{array}{c}\text { Traitement } \\
\text { phytosani- } \\
\text { taire } \\
(D T / h a)\end{array}$ & $\begin{array}{l}\text { Fertilisation } \\
\text { (DT/ha) }\end{array}$ & $\begin{array}{c}\text { Semences } \\
\text { (DT/ha) }\end{array}$ & Divers & $\begin{array}{c}\text { Main } \\
\text { d'œuvre } \\
\text { (DT/ha) }\end{array}$ & $\begin{array}{c}\text { Total } \\
\text { charges } \\
\text { variables } \\
(D T / h a)\end{array}$ \\
\hline \multirow{5}{*}{$\begin{array}{l}\text { Strate } 1 \\
(1-5 \text { ha) }\end{array}$} & $\mathbf{N}$ & 81 & 81 & 81 & 81 & 81 & 81 & 81 \\
\hline & Moyenne & 501,9 & 137,5 & 208,7 & 163,0 & 147,8 & 53,3 & 1148,6 \\
\hline & Minimum & 305,3 & 0,0 & 0,0 & 105,0 & 72,0 & 37,5 & 642,6 \\
\hline & Maximum & 850,5 & 270,0 & 373,8 & 300,0 & 315,0 & 195,0 & 1763,9 \\
\hline & Ecart-type & 99,7 & 69,0 & 65,3 & 48,7 & 69,6 & 22,7 & 223,9 \\
\hline \multirow{5}{*}{$\begin{array}{l}\text { Strate } 2 \\
\text { (5-10ha) }\end{array}$} & $\mathbf{N}$ & 40 & 40 & 40 & 40 & 40 & 40 & 40 \\
\hline & Moyenne & 481,3 & 125,5 & 171,9 & 153,2 & 147,0 & 54,2 & 1066,9 \\
\hline & Minimum & 332,8 & 0,0 & 0,0 & 65,3 & 84,0 & 33,8 & 675,7 \\
\hline & Maximum & 664,5 & 262,7 & 292,5 & 300,0 & 315,0 & 123,0 & 1501,9 \\
\hline & Ecart-type & 80,9 & 58,8 & 66,4 & 49,0 & 67,1 & 18,6 & 196,3 \\
\hline \multirow{5}{*}{$\begin{array}{l}\text { Strate 3 } \\
(10-50 \mathrm{ha})\end{array}$} & $\mathbf{N}$ & 26 & 26 & 26 & 26 & 26 & 26 & 26 \\
\hline & Moyenne & 505,8 & 129,6 & 210,7 & 159,5 & 124,4 & 47,5 & 1125,3 \\
\hline & Minimum & 376,5 & 0,0 & 0,0 & 130,5 & 90,0 & 45,0 & 585,3 \\
\hline & Maximum & 727,9 & 262,7 & 324,0 & 414,0 & 315,0 & 60,0 & 1707,4 \\
\hline & Ecart-type & 91,9 & 73,1 & 73,9 & 60,6 & 56,6 & 3,2 & 253,6 \\
\hline \multirow{5}{*}{$\begin{array}{l}\text { Strate } 4 \\
\text { (Sup à } \\
50 \mathrm{ha})\end{array}$} & $\mathbf{N}$ & 85 & 85 & 85 & 85 & 85 & 85 & 85 \\
\hline & Moyenne & 538,2 & 170,2 & 233,8 & 159,0 & 104,0 & 48,7 & 1196,7 \\
\hline & Minimum & 389,3 & 0,0 & 0,0 & 120,0 & 72,0 & 32,0 & 624,4 \\
\hline & Maximum & 770,3 & 337,5 & 556,5 & 300,0 & 315,0 & 93,0 & 1634,3 \\
\hline & Ecart-type & 97,8 & 81,6 & 83,6 & 41,5 & 32,7 & 9,4 & 214,9 \\
\hline \multirow{4}{*}{ Total } & Moyenne & 512,1 & 146,4 & 211,7 & 159,5 & 129,1 & 51,1 & 1149,5 \\
\hline & Minimum & 305,3 & 0,0 & 0,0 & 65,3 & 72,0 & 32,0 & 585,3 \\
\hline & Maximum & 850,5 & 337,5 & 556,5 & 414,0 & 315,0 & 195,0 & 1763,9 \\
\hline & Ecart-type & 96,9 & 74,6 & 76,2 & 47,6 & 59,9 & 16,6 & 222,9 \\
\hline
\end{tabular}

Source : Nos calculs à partir de notre enquête.

tillon d'environ $585 \mathrm{DT} /$ ha à $1764 \mathrm{DT} /$ ha soit, en moyenne 1149DT/ha. La strate 4 présente la valeur la plus élevée soit de l'ordre de 1197 DT/ ha contre 1149DT/ha, $1067 \mathrm{DT} /$ ha et $1125 \mathrm{DT} / \mathrm{ha}$ respectivement pour les strates 1,2 et 3 .

L'activité céréalière est l'activité agricole la plus mécanisée. En effet, la charge de mécanisation occupe la première place en termes de valeur pour les différentes tailles d'exploitation.
Les charges de mécanisation oscillent entre un minimum de 305DT/ha et un maximum de 851 DT/ha. La préparation du sol seule représente $12 \%$ du total des charges variables.

En termes d'importance des charges par rapport au total des charges variables, le Tableau 3 permet de classer ces valeurs par ordre d'importance comme suit : la mécanisation, la fertilisation, les traitements phytosanitaires (trai- 
Tableau 3 - Structure des charges variables pour la production du blé dur.

\begin{tabular}{|lccccccc|}
\hline Unité \% & Mécanisation & Fertilisation & $\begin{array}{c}\text { Traitement } \\
\text { phytosanitaire }\end{array}$ & Semences & Divers & $\begin{array}{c}\text { Main } \\
\text { d'œeuve }\end{array}$ & $\begin{array}{c}\text { Total } \\
\text { charges } \\
\text { variables }\end{array}$ \\
\hline $\begin{array}{l}\text { Strate } 1 \\
(1-5 \text { ha })\end{array}$ & 43,7 & 18 & 12 & 13 & 14 & 5 & 100 \\
\hline $\begin{array}{l}\text { Strate 2 } \\
(5-10 \mathrm{ha})\end{array}$ & 45 & 16 & 12 & 14 & 14 & 5 & 100 \\
\hline $\begin{array}{l}\text { Strate 3 } \\
(10-50 \mathrm{ha})\end{array}$ & 45 & 19 & 12 & 11 & 14 & 4 & 100 \\
\hline $\begin{array}{l}\text { Strate } 4 \\
\text { (Sup à } \\
50 \text { ha) }\end{array}$ & 45 & 20 & 14 & 9 & 13 & 4 & 100 \\
\hline Total & 45 & 18 & 13 & 11 & 14 & 4 & 100 \\
\hline
\end{tabular}

Source : Nos calculs à partir de notre enquête.

tement fongique et désherbage), les semences, les charges diverses et la main d'œuvre avec des taux respectifs de $45 \%, 18 \%, 13 \%, 11 \%$, $14 \%$ et $4 \%$. Ce même tableau indique que les charges de production présentent des taux respectifs comparables pour les différentes strates de superficie.

\subsubsection{Charges fixes}

Pour surmonter le problème de répartition des charges fixes entre les cultures pour une même exploitation et afin d'homogénéiser les données et de réduire l'effet du capital fixe et/ ou cumulé, les frais d'amortissement ne sont pas comptabilisés dans le calcul de ces charges. L'hypothèse dans ce cas est de considérer que toutes les exploitations ont recours aux services extérieurs. Le matériel utilisé est considéré comme loué par l'exploitant.

La somme de charges fixes varie de près de 566DT/ha à $1138 \mathrm{DT} / \mathrm{ha}$, soit en moyenne 832DT/ha pour l'ensemble des exploitations.

Les charges financières sont en moyenne de 168DT/ha et varient de $68 \mathrm{DT} /$ ha à $148 \mathrm{DT} / \mathrm{ha}$. Quant à la valeur locative de la terre, elle dépend de la texture du sol et de la zone de production liée à la pluviométrie. Celle-ci varie de 250 DT/ ha à $350 \mathrm{DT} / \mathrm{ha}$ et représente, de ce fait, une moyenne de $290 \mathrm{DT} / \mathrm{ha}$.

Les frais d'assurance sont considérés comme des frais facultatifs. Généralement, ces frais sont liés aux types de financement. En effet, pour les crédits agricoles et notamment les crédits de campagne, les assurances sont toujours exigées par la Banque Nationale Agricole qui est généralement la seule institution financière chargée $\mathrm{du}$ financement du secteur agricole. Ces charges sont en moyenne d'environ 8DT/ha et varient de 2 à $17 \mathrm{DT} / \mathrm{h}$.

Les charges financières ont été prises en considération dans le calcul des charges fixes. Ces frais ont été estimés aux taux d'intérêt du capital investi, soit de $8,5 \%$ des charges totales. Ce taux est jugé, par certains agriculteurs, relativement élevé, comparé au taux accordé au secteur du bâtiment qui est de l'ordre de $7 \%$.

Il en ressort du Tableau 4 que la valeur locative de la terre représente près de la moitié du total des charges fixes. Les frais de gestion, les frais financiers et les assurances occupent en moyenne $19 \%, 27 \%$ et $1 \%$ respectivement des charges fixes. Les frais de gestion couvrent les frais d'approvisionnement, le déplacement et le contrôle. Ils sont souvent sous-estimés par les agriculteurs et sont confondus avec les frais de la main d'œuvre familiale. Pour l'ensemble de ces frais, la part moyenne présente une différence minime entre les strates d'exploitations.

Le Tableau 5 montre que les charges totales de production du blé dur par hectare sont en moyenne de 1982 DT/ha, variant de 1219 DT/ ha à $2753 \mathrm{DT} / \mathrm{ha}$. Cette variation dépend généralement du degré d'intensification et de la technique de production adoptée par les agriculteurs. 
Tableau 4 - Total des charges fixes pour la production du blé dur par strate de superficie.

\begin{tabular}{|c|c|c|c|c|c|c|}
\hline Strates & & $\begin{array}{c}\text { Frais } \\
\text { d'assurance } \\
\text { (DT/ha) }\end{array}$ & $\begin{array}{c}\text { Valeur locative } \\
\text { de la terre } \\
\text { (DT/ha) }\end{array}$ & $\begin{array}{c}\text { Charges } \\
\text { financières } \\
(D T / h a)\end{array}$ & $\begin{array}{l}\text { Frais de } \\
\text { gestion } \\
\text { (DT/ha) }\end{array}$ & $\begin{array}{l}\text { Charges } \\
\text { fixes } \\
\text { (DT/ha) }\end{array}$ \\
\hline \multirow{5}{*}{$\begin{array}{l}\text { Strate } 1 \\
(1-5 \text { ha) }\end{array}$} & $\mathrm{N}$ & 81 & 81 & 81 & 81 & 81 \\
\hline & Moyenne & 6,927 & 256,79 & 102,253 & 138,524 & 756,793 \\
\hline & Minimum & 2,46 & 250 & 67,84 & 49,2 & 576,99 \\
\hline & Maximum & 15,04 & 300 & 147,59 & 300,8 & 1075,3 \\
\hline & Ecart-type & 2,773 & 17,236 & 15,087 & 55,45 & 102,9 \\
\hline \multirow{5}{*}{$\begin{array}{l}\text { Strate 2 } \\
(5-10 \mathrm{ha})\end{array}$} & $\mathrm{N}$ & 40 & 40 & 40 & 40 & 40 \\
\hline & Moyenne & 6,887 & 263,75 & 97,504 & 137,728 & 758,8 \\
\hline & Minimum & 1,85 & 250 & 75,05 & 36,9 & 566 \\
\hline & Maximum & 15,75 & 350 & 125,13 & 315 & 1087,53 \\
\hline & Ecart-type & 3,167 & 25,287 & 13,349 & 63,35 & 126,925 \\
\hline \multirow{5}{*}{$\begin{array}{l}\text { Strate 3 } \\
\text { (10-50ha) }\end{array}$} & $\mathrm{N}$ & 26 & 26 & 26 & 26 & 26 \\
\hline & Moyenne & 7,519 & 288,462 & 103,867 & 150,364 & 825,25 \\
\hline & Minimum & 3,55 & 250 & 69,02 & 70,95 & 625,81 \\
\hline & Maximum & 17,19 & 350 & 143,83 & 343,71 & 1138,18 \\
\hline & Ecart-type & 3,119 & 38,23 & 19,301 & 62,38 & 137,939 \\
\hline \multirow{5}{*}{$\begin{array}{l}\text { Strate } 4 \\
\text { (Sup à 50ha) }\end{array}$} & $\mathrm{N}$ & 85 & 85 & 85 & 85 & 85 \\
\hline & Moyenne & 8,551 & 334,71 & 113,252 & 171 & 941,308 \\
\hline & Minimum & 2,65 & 250 & 74,04 & 53 & 580,85 \\
\hline & Maximum & 13,2 & 350 & 143,95 & 264 & 1118,480 \\
\hline & Ecart-type & 2,121 & 30,883 & 15,706 & 42,423 & 104 \\
\hline \multirow{5}{*}{ Total } & $\mathrm{N}$ & 232 & 232 & 232 & 232 & 232 \\
\hline & Moyenne & 8,32 & 290,086 & 168,46 & 257,612 & 832,424 \\
\hline & Minimum & 1,85 & 250 & 67,84 & 36,9 & 566,31 \\
\hline & Maximum & 17,19 & 350 & 147,59 & 343,71 & 1138,4 \\
\hline & Ecart-type & 2,762 & 44,244 & 16,616 & 55,226 & 140,27 \\
\hline
\end{tabular}

Source : Nos calculs à partir de notre enquête.

En effet, ces charges varient entre les strates de superficie. La valeur moyenne la plus faible est repérée dans la strate 2 , soit $1826 \mathrm{DT} /$ ha contre $2138 \mathrm{DT} /$ ha pour la strate 4 .

Le niveau de production est estimé à partir des déclarations des agriculteurs enquêtés. Le rendement en grain varie de $6 \mathrm{q} / \mathrm{ha}$ à environ $57 \mathrm{q} / \mathrm{ha}$ avec une moyenne de $24 \mathrm{q} / \mathrm{ha}$. Cette moyenne est proche de la moyenne nationale durant les campagnes antérieures. Il est à remarquer que le rendement est nettement meil- leur pour la strate 4 . Toutefois, il est le même pour les strates 1 et 2 .

Le coût unitaire de production d'un quintal de blé dur produit dans les régions subhumides varie de 30,5DT/q à 239DT/q avec une moyenne de 78DT/q. Le coût moyen le plus faible est observé dans la strate 4 et le plus élevé est repéré dans la strate 1et 2 .

Cependant, malgré ce coût élevé, il reste au-dessous du prix à la production fixé par l'Etat à savoir 80 DT/q. 
Tableau 5 - Total des charges, rendement en grain et coût unitaire de production du blé dur par strate de superficie.

\begin{tabular}{|c|c|c|c|c|}
\hline & & $\begin{array}{c}\text { Charges } \\
\text { totales } \\
(D T / h a)\end{array}$ & $\begin{array}{l}\text { Rendement } \\
\text { grain } \\
(q)\end{array}$ & $\begin{array}{c}\text { Coût } \\
\text { Unitaire } \\
(D T / q)\end{array}$ \\
\hline \multirow{4}{*}{$\begin{array}{l}\text { Strate } 1 \\
(1-5 \mathrm{ha})\end{array}$} & Moyenne & 1905,3 & 22,5 & 83,3 \\
\hline & Minimum & 1218,6 & 8,0 & 33,1 \\
\hline & Maximum & 2740,0 & 54,0 & 199,9 \\
\hline & Ecart-type & 281,8 & 9,8 & 35,1 \\
\hline \multirow{4}{*}{$\begin{array}{c}\text { Strate } 2 \\
(5-10 \mathrm{ha})\end{array}$} & Moyenne & 1825,7 & 22,5 & 84,3 \\
\hline & Minimum & 1462,3 & 6,0 & 30,5 \\
\hline & Maximum & 2407,0 & 55,0 & 239,0 \\
\hline & Ecart-type & 258,4 & 11,1 & 45,5 \\
\hline \multirow{4}{*}{$\begin{array}{l}\text { Strate } 3 \\
(10-50 \mathrm{ha})\end{array}$} & Moyenne & 1950,6 & 24,5 & 77,7 \\
\hline & Minimum & 1340,4 & 11,0 & 31,7 \\
\hline & Maximum & 2587,3 & 57,1 & 153,0 \\
\hline & Ecart-type & 332,1 & 10,7 & 31,9 \\
\hline \multirow{4}{*}{$\begin{array}{l}\text { Strate } 4 \\
\text { (Sup à } 50 \text { ha) }\end{array}$} & Moyenne & 2138,0 & 26,7 & 70,2 \\
\hline & Minimum & 1420,1 & 8,0 & 34,9 \\
\hline & Maximum & 2752,7 & 42,0 & 178,6 \\
\hline & Ecart-type & 289,8 & 6,7 & 22,7 \\
\hline \multirow{4}{*}{ Total } & Moyenne & 1981,9 & 24,2 & 78,0 \\
\hline & Minimum & 1218,6 & 6,0 & 30,5 \\
\hline & Maximum & 2752,7 & 57,1 & 239,0 \\
\hline & Ecart-type & 310,789 & 9,304 & 33,357 \\
\hline
\end{tabular}

Source : Nos calculs à partir de notre enquête.

4.2. Produits bruts et résultats d'exploitation

Bien que les prix des céréales est annoncé au début de la campagne agricole afin d'encourager les agriculteurs à la production du blé et des céréales, il existe en général une variation importante entre le prix annoncé et le prix de vente. En effet, le prix du blé payé aux agriculteurs est différent du prix déclaré par l'Office des Céréales au début de la campagne. Ce prix pour l'année 2018-2019 est de 70 DT/q majoré par une prime de collecte de 15 DT/q. Cependant, le prix réellement reçu par les céréaliers varie de 67DT/q à $83 \mathrm{DT} / \mathrm{q}$. Ce dernier dépend de la période de vente et de la qualité du blé récolté. Toutefois, cette qualité dépend de plu- sieurs facteurs à savoir : (i) le poids de 1000 grains ou le poids spécifique ; (ii) l'impureté du blé $(\%$ des déchets, $\%$ des grains maigres ou cassées) ; (iii) la contamination des graines essentiellement par le métadinage ; (iv) et enfin l'évaluation du collecteur.

Au cours des dernières campagnes, un circuit de commerce parallèle est apparu. Ce nouveau circuit a surtout intéressé les agriculteurs endettés afin d'éviter le prélèvement direct par la banque et le retard de payement. L'autre argument déclaré par les agriculteurs justifiant le recours à ce circuit est surtout la sous-estimation du prix d'achat par les collecteurs certifiés (silos) et ce malgré l'existence d'une commission de droiture auprès des CRDA. 
Tableau 6 - Produit brut, marge brute et marge nette par strate de superficie d'exploitation.

\begin{tabular}{|c|c|c|c|c|}
\hline & & $\begin{array}{c}\text { Produit brut } \\
\text { (DT/ha) }\end{array}$ & $\begin{array}{c}\text { Marge brute } \\
\text { (DT/ha) }\end{array}$ & $\begin{array}{c}\text { Marge nette } \\
\text { (DT/ha) }\end{array}$ \\
\hline \multirow{4}{*}{$\begin{array}{l}\text { Strate1 } \\
(1-5 \text { ha })\end{array}$} & Moyenne & 2077,9 & 929,3 & 172,5 \\
\hline & Minimum & 738,0 & $-387,6$ & $-991,5$ \\
\hline & Maximum & 4512,0 & 3418,0 & 2422,3 \\
\hline & Ecart-type & 831,7 & 814,8 & 729,8 \\
\hline \multirow{4}{*}{$\begin{array}{l}\text { Strate2 } \\
(5-10 \mathrm{ha})\end{array}$} & Moyenne & 2065,9 & 999,0 & 240,2 \\
\hline & Minimum & 553,5 & $-400,0$ & $-981,1$ \\
\hline & Maximum & 4725,0 & 3760,9 & 2673,3 \\
\hline & Ecart-type & 950,2 & 949,0 & 837,3 \\
\hline \multirow{4}{*}{$\begin{array}{l}\text { Strate3 } \\
\text { (10-50ha) }\end{array}$} & Moyenne & 2255,5 & 1130,2 & 304,9 \\
\hline & Minimum & 1064,3 & $-146,2$ & $-882,1$ \\
\hline & Maximum & 5155,7 & 4137,6 & 2999,4 \\
\hline & Ecart-type & 935,7 & 987,5 & 899,6 \\
\hline \multirow{4}{*}{$\begin{array}{l}\text { Strate4 } \\
\text { (Sup à 50ha) }\end{array}$} & Moyenne & 2565,0 & 1368,3 & 427,0 \\
\hline & Minimum & 795,0 & $-158,6$ & $-976,4$ \\
\hline & Maximum & 3960,0 & 2723,1 & 1606,1 \\
\hline & Ecart-type & 636,4 & 597,9 & 535,2 \\
\hline \multirow{4}{*}{ Total } & Moyenne & 2274,2 & 1124,7 & 292,3 \\
\hline & Minimum & 553,5 & $-400,0$ & $-991,5$ \\
\hline & Maximum & 5155,7 & 4137,6 & 2999,4 \\
\hline & Ecart-type & 828,39 & 809,582 & 711,85 \\
\hline
\end{tabular}

Source : Nos calculs à partir de notre enquête.

Le produit brut dépend du rendement en grain et en paille ainsi que de leurs prix. Les calculs réalisés montrent que le produit brut enregistre une variation importante par hectare allant de 554DT/ha à 5156DT/ha, soit en moyenne 2274DT/ha pour l'ensemble des exploitations enquêtées. Le produit brut pour les strates de superficie 1, 2, 3 et 4 est en moyenne de 2078DT/ha, 2067DT/ha 2256DT/ha et $2565 \mathrm{DT} /$ ha respectivement.

Le Tableau 6 indique que la marge brute varie de $-400 \mathrm{DT} /$ ha à $4136 \mathrm{DT} / \mathrm{ha}$. La moyenne estimée est de 1125DT/ha pour le total des exploitations enquêtées. Les marges brutes moyennes pour les strates sont différentes. Elles sont plus élevées pour la strate 4 (environ 1368 DT/ha), suivi de la strate 3 (1130 DT/ha), de la strate 2
(999 DT/ha) et de la strate 1 (929 DT/ha). Cette fluctuation résulte de l'écart du rendement observé entre les strates, du prix du blé dur et des charges d'exploitation ou de production.

\subsection{Rentabilité et productivité des exploitations}

\subsubsection{Seuil de rentabilité de la culture de blé dur}

Le seuil de rentabilité d'une activité donnée est identifié par l'intersection et/ou l'égalité entre la marge brute et les charges fixes (Boubaker, 2000). A partir de ce seuil, l'agriculteur réalise une marge nette positive. De même, ce seuil est atteint si les charges totales sont égales 
Figure 5 - Seuil de rentabilité pour les exploitations céréalières.

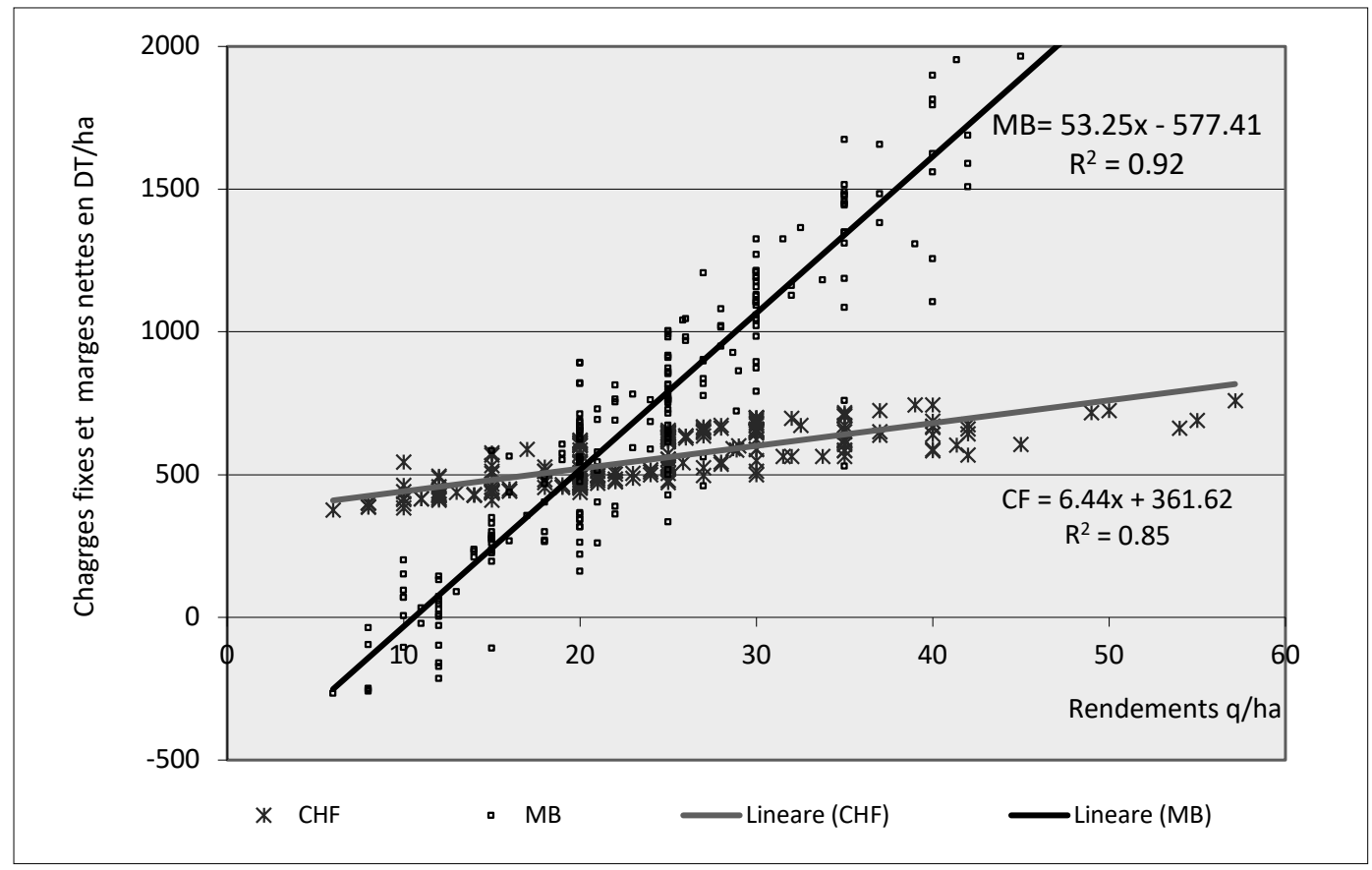

Source : Notre enquête.

au produit brut et dans ce cas-ci les marges nettes s'annulent.

$$
\mathrm{Y}^{*}=(\mathrm{CV}+\mathrm{CF}) / \mathrm{P} \text { ou } \mathrm{MN}=0
$$

La Figure 5 montre la répartition des agriculteurs en fonction des rendements réalisés, des charges fixes et des marges assurées (brutes) par la culture du blé dur. Cette même figure indique que l'intersection des deux courbes, charges fixes et marges brutes, se fait au point « seuil de rentabilité ». Cette résolution graphique peut être traduite par les équations obtenues par les courbes de tendances.

\section{Charges fixes $=$ marge brute}

D'où le seuil de rentabilité est $x=20,2 q /$ ha.

De la même logique, le seuil de rentabilité des grandes exploitations est de 20,7 q/ha. Ce seuil n'est atteint que par $67,9 \%$ des agriculteurs de la strate 1 (0-5 ha), 63\% de la strate 2 (5-10 ha), $62 \%$ de la strate 3 (10-50 ha) et $90,6 \%$ de la strate 4 (supérieur à 50 ha).

De ce fait, le seuil de rentabilité est presque le même aussi bien pour les petites que les grandes exploitations, ce qui valide l'homogénéité entre les tailles d'exploitations. Cette situation est expliquée par l'équité de l'accessibilité au marché des facteurs de production des céréales, notamment le blé dur. Ainsi, la taille d'exploitation n'est pas un facteur déterminant de la rentabilité du blé dur.

\subsubsection{Productivité totale des facteurs de pro- duction du blé dur}

La PTF de production du blé dur calculée pour notre échantillon est en moyenne de 2 , variant de 0,58 à 5,66. Par taille d'exploitation, elle est en moyenne de 1,85 pour les exploitations dont la superficie ne dépasse pas les 5 ha, de 1,99 pour les superficies entre 5 et 10 ha et elle est presque la même pour les exploitations dont les superficies dépassent 10 ha. Par contre, le maximum de productivité est observé pour les trois premières strates. Par conséquent, une dépense d'un dinar dans les charges variables de production engendre un produit brut de 1,85 DT/ ha pour la strate 1, alors que ce montant est de 2,18 DT/ha pour les exploitations supérieures à 
Tableau 6 - PTF par strate de superficies.

\begin{tabular}{|lccccc|}
\hline Superficie & $N$ & Moyenne & Maximum & Minimum & Écart-type \\
\hline 1ha -5ha & 81 & 1,85 & 4,26 & 0,66 & 0,774 \\
\hline Entre 5 et 10 ha & 40 & 1,99 & 5,66 & 0,58 & 1,028 \\
\hline Entre 10-50ha & 26 & 2,12 & 5,06 & 0,88 & 1,093 \\
\hline Supérieur à 50 ha & 85 & 2,18 & 3,32 & 0,87 & 0,563 \\
\hline Total & 232 & 2,025 & 5,66 & 0,58 & 0,807 \\
\hline
\end{tabular}

Source : Nos calculs à partir de notre enquête.

50 ha. Ceci confirme l'effet positif de la taille sur la productivité totale des facteurs de l'exploitation. Ce résultat est en harmonie avec le résultat de Grami et Ben Rejeb (2015) qui ont montré la relation positive entre le rendement et la taille de l'exploitation dans la région de Béja. L'analyse ANOVA montre que la différence de la PTF entre les strates de superficie est statistiquement significative au seuil de $10 \%$. Toutefois, le test Lsd montre que cette différence est détectée seulement entre grande (supérieure à 50 ha) et petite taille (inférieure à10 ha).

\subsection{Déterminants de la productivité}

Le Tableau 7 présente les facteurs qui semblent expliquer la PTF du blé dur. Plusieurs tests ont été effectués pour identifier les déterminants de la productivité à savoir la taille de l'exploitation (la superficie totale), la rotation $(1=$ oui $0=$ non), les variétés (nouvelles $=1$ anciennes $=0$ ), les types de semences (sélectionnées, ordinaires et autoproduites), l'investissement réalisé sur l'exploitation ( $1=1$ 'exploitant a réalisé des projets d'investissements, $0=$ non) et le morcellement (nombre de parcelles exploitées).

L'ensemble de ces variables retenues explique $39 \%$ de la variabilité de la PTF. Il ressort que la taille de l'exploitation et la rotation et/ou le précédent cultural ont un effet positif sur la productivité de la culture de blé dans les régions subhumides. Alors que, l'utilisation des variétés nouvelles affecte négativement la productivité du blé dur à l'instar des variétés Karim et Razeg par rapport à Maali et Naser, notamment pour le cas de cette année sèche, vu que ces deux dernières sont plus exigeantes en eau (pluviométrie). Cet effet significatif de la variété est prouvé par le travail de Slama et al. (2005).

Tableau 7 - Déterminant de la productivité totale des facteurs de production du blé dur.

\begin{tabular}{|l|c|c|c|}
\hline Variable & Coefficient & Erreur. Std & t-Statistic \\
\hline C & $1,353^{*}$ & 0,840 & 1,611 \\
\hline Taille de l'exploitation & $0,014^{*}$ & 0,008 & 1,782 \\
\hline Rotation & $0,196^{* *}$ & 0,063 & 3,123 \\
\hline Variétés utilisées & $-0,114^{*}$ & 0,060 & $-1,906$ \\
\hline Types de semences & 0,012 & 0,014 & 0,886 \\
\hline $\begin{array}{l}\text { Investissement à } \\
\text { l'exploitation }\end{array}$ & $-0,566^{* *}$ & 0,139 & $-4,056$ \\
\hline Morcellement & $-0,006$ & 0,017 & $-0,342$ \\
\hline R-carré & 0,39 & & \\
\hline
\end{tabular}

** significative au niveau $1 \%$; * significative au niveau $10 \%$.

Source: Nos calculs à partir de notre enquête. 
La réalisation des projets d'investissement sur l'exploitation a un effet négatif sur la PTF dans notre cas par contre plusieurs études ont montré l'inverse (Doukkali et Guèdègbé, 2018). Ceci peut être expliquée par le fait que l'exploitant a investi dans d'autres activités autre que la production des céréales. Par conséquent, il s'intéresse à ces nouvelles affaires introduites et non à l'amélioration de la productivité des céréales. Cette contradiction est cohérente avec le résultat de Cette et Szpiro (1990) qui explique que l'investissement enregistré n'est pas en rapport avec le progrès technique. Il s'agit d'un investissement isolé comme a été nommé par Cabral et al. (2017).

\section{Conclusion générale}

Le rendement n'est pas considéré comme le seul facteur déterminant de la rentabilité de la culture de blé dur. L'analyse des prix des facteurs et des produits ainsi que la productivité totale des facteurs constituent des éléments importants dans l'examen de la performance économique des exploitations.

L'analyse empirique de ce travail a été réalisée en tenant compte des strates de superficie, indicateur habituellement utilisé par les pouvoirs publics pour l'élaboration des politiques agricoles.

Les résultats obtenus montrent que les charges totales de production du blé dur varient en fonction de la taille de l'exploitation réalisant un écart moyen de 829DT/ha. Par strate de superficie, la valeur la plus importante des charges est repérée pour les exploitations ayant des superficies importantes (supérieure à $50 \mathrm{ha}$ ), par contre elles sont les mêmes pour la strate 1 (1-5 ha) et la strate 2 (5-10 ha) avec une légère différence marquée pour la troisième strate (10-50 ha). Ce diagnostic par strate montre que plus la taille de l'exploitation augmente plus les charges par hectare s'amplifient. Par contre les faibles marges brutes sont repérées pour les petites exploitations dont la taille est inférieure à 10 ha.

Parallèlement, l'analyse de la productivité totale des facteurs fait ressortir que la productivité totale des facteurs est en moyenne de l'ordre de 2. Par strate de superficie, la meilleure productivité moyenne est observée au niveau des superficies dont la taille dépasse les 10 ha.
L'examen des déterminants de la productivité indique que la productivité est affectée négativement par les variétés exigeantes en eau et les investissements réalisés à l'exploitation et positivement par la rotation des cultures.

Finalement, près de $25 \%$ des exploitations sont au-dessous de seuil de la rentabilité. De ce fait, la production du blé dur est menacée. Des mesures politiques plus adaptées à la taille de la production seront nécessaires.

Ainsi, il est recommandé d'encourager la rotation des cultures à travers une vulgarisation intensive et efficace auprès des agriculteurs. En plus, la production et la conservation des anciennes variétés doivent être encouragées moyennant des incitations économiques aux agriculteurs qui les utilisent. Ces variétés ont montré leur adaptation aux conditions climatiques et édaphiques sévères qui caractérisent notre milieu de production. Celles-ci soutiennent la durabilité du système de production à base des céréales. En plus, la préservation de la taille des exploitations par une politique foncière qui limiterait le morcellement des parcelles et l'encouragement des agriculteurs de s'organiser en des coopératives de production, est une urgence et un enjeu fort pour le maintien d'une céréaliculture durable.

\section{Remerciements}

Les auteurs tiennent à remercier vivement les referees anonymes pour leurs commentaires constructifs et leurs suggestions qui ont permis l'aboutissement de ce papier dans sa présente structure.

\section{Références bibliographiques}

Albouchi L., 2006. Gestion de l'eau en Tunisie: d'une politique de mobilisation à une politique de réallocation de la ressource selon sa valorisation économique : cas du bassin versant de Merguellil, Tunisie centrale. Thèse de doctorat, Faculté des sciences économiques de Montpellier, $232 \mathrm{p}$.

Bachta M.S., El Abbassi M., Ferktaji R., 2001. Compétitivité des produits agricoles tunisiens exportés et coûts en ressources naturelles. In : Laajimi A., Arfa L. (dir.), Le futur des échanges agro-alimentaires dans le bassin méditerranéen : Les enjeux de la mondialisation et les défis de la compétitivité. 
Zaragoza : CIHEAM, pp. 101-111 (Cahiers Options Méditerranéennes, 57).

Bamidele F.S., Babatunde R.O., Rasheed A., 2008. Productivity analysis of cassava-based production systems in the Guinea Savannah: Case study of Kwara State, Nigeria. American-Eurasian Journal of Scientific Research, 3(1): 33-39.

Ben Ammar B., 1995. La politique céréalière en Tunisie. Stratégie de la filière céréalière en Tunisie. Communication au séminaire CIHEAM-IRESA, Tunis.

Boubaker M., 2000. La comptabilité de gestion. Tunis : Edition C.L.E., 235 p.

Boughanmi H., 1995. Les principaux volets des politiques agricoles en Tunisie : évolution, analyse et performances agricoles. In : Allaya M. (dir.), Les agricultures maghrébines à l'aube de l'an 2000. Montpellier : CIHEAM, pp. 127-138 (Options Méditerranéennes : Série B. Etudes et Recherches, 14).

Cabral F.J., Cissé F., Diagne A., 2017. Croissance agricole, option d'investissement et réduction de la pauvreté au Sénégal. Forum de Vulgarisation des résultats de la recherche économique et sociale.

Cette G., Szpiro D., 1990. L'influence de l'investissement sur la productivité via le progrès technique. In : La productivité : Tendances et facteurs explicatifs. Economie et statistique, 237-238: 87-98.

Chebil A., Frija A., Thabet C., 2015. Economic efficiency measures and its determinants for irrigated wheat farms in Tunisia: a DEA approach. New Medit, 14(2): 32-38.

Desbois D., 2002. Coûts de production agricole : Comparaison de deux méthodes d'estimation sur la base du RICA. In : Insee-Méthodes : Actes des Journées de Méthodologie Statistique 2002. Montrouge : Institut national de la statistique et des études économiques.

Desbois D., 2006. Méthodologie d'estimation des coûts de production agricole : comparaison de deux méthodes sur la base du RICA. La revue MODULAD, 45-72 (hal-02656166).

Desbois D., Legris B., 2007. Prix et coût de production de six grandes cultures : blé, maïs, colza, tournesol, betterave et pomme de terre. In : L'agriculture, nouveau défis. Edition 2007. Montrouge : Institut national de la statistique et des études économiques.

Dhehibi B., Bahri H., Annabi M., 2012. Input and output technical efficiency and total factor productivity of wheat production in Tunisia. African Journal of Agricultural and Resource Economics, 7(1): 1-18.

Dhehibi B., Lachaal L., 2004. Evidence empirique de la croissance de la productivité de l'agricul- ture tunisienne : 1961-2000, 10 p. https://www. afdb.org/sites/default/files/documents/publications/09484303-fr-empirical-evidence-of-productivity-growth-in-tunisian-agriculture.pdf.

Doukkali M.R., Guèdègbé T., 2018. Contribution des facteurs et de la productivité globale des facteurs à la croissance agricole au Maroc: une évaluation comparée intersectorielle et inter pays. Research Paper \& Policy papers, 1809, Policy Center for the New South.

FAO (Food and Agricultural Organization), 2005. Analyse de financement du secteur agricole en Tunisie. Revue sectorielle.

FAO (Food and Agricultural Organization), 2013. FAOSTAT: www.fao.org/faostat/fr.

FAO (Food and Agricultural Organization), 2020. World Food Situation. http://www.fao.org/worldfoodsituation $/ \mathrm{csdb} / \mathrm{fr} /$.

Filippini M., 2014. Coût de production, efficacité et compétitivité à court terme des entreprises hydrauliques suisses. Centre d'énergie politique et économique, ETH, Zurich. Faculté des sciences économiques, Università della Svizzera Italiana. Journée de l'énergie 2014.

Garten, Dubois, 2006. Le coût de production de la fèverole chez nos concurrents.

Gharbi F., El Fahem M., Lahsoumi F., Rached Z., 2002. Analyse de la rentabilité de production des semences de la pomme de terre. BASE.

Grami D., Ben Rejeb J., 2015. L'impact des changements climatiques sur le rendement de la céréaliculture dans la région du Nord-Ouest de la Tunisie (Béja). New Medit, 14(4): 36-41.

INS (Institut National de la statistique), 2010-2015. Enquête nationale sur le budget, la consommation et le niveau de vie des ménages. Tunis, Tunisie.

Karray B., Louzi A., Sahnoun A. 2000. Estimation du cout de production d'olives a huile en Tunisie: application de la methode de cout constate aux exploitations privées dans la region de Sfax. New Medit, 11(4): 11-18.

Khaldi S.R., Zekri S., 2002. Etude des légumineuses alimentaires dans les systèmes de production du nord de la Tunisie : situation actuelle et possibilité de développement. Tunis : Ministère de l'Agriculture - GTZ. Lasram A., Dellagi H., Masmoudi, M.M., Ben Mechlia N., 2015. Productivité de l'eau du blé dur irrigué face à la variabilité climatique. New Medit, 14(1): 61-66.

MARHP (Ministère de l'Agriculture des Ressources Hydrauliques et de la Pêche), 2006. Revue du secteur agricole : Etudes sur le financement du secteur agricole. DGEDA/CNEA. 
MARHP (Ministère de l'Agriculture des Ressources Hydrauliques et de la Pêche), 2007. XI ime Plan de développement 2007-2011. DGEDA.

MARHP (Ministère de l'Agriculture des Ressources Hydrauliques et de la Pêche), 2012, 2015. Budgets économiques. DGEDA/ONAGRI. http://www.onagri.tn/.

Mbam B.N., Edeh H.O., 2011. Determinants of farm productivity among small holder rice farmers in Anambra State, Nigeria. Journal of Animal \& Plant Sciences, 9(3): 1187-1191.

Nkamleu G.B., 2004. L'échec de la croissance de la productivité agricole en Afrique francophone. Economie Rurale, 279(1) : 53-65.
Office des céréales, 2012. Sous direction de contrôle. Office des céréales Direction générale. Rapport d'activité annuel - 2012 à 2016.

Rached Z., 2008. Analyse comparative des coûts de production et de l'efficacité technique des dattes biologiques : cas de la région de Hezoua. Mémoire de Mastère. Institut National Agronomique de Tunisie, $140 \mathrm{p}$.

Slama A., Ben Salem M., Ben Naceur M., Zid E., 2005. Les céréales en Tunisie: production, effet de la sècheresse et mécanismes de résistance. Sècheresse, 16(3) : 225-229. 
